\title{
A Bench Scale Investigation of Pump-Ejector System at Simultaneous Water and Gas Injection
}

\author{
S. Karabaev ${ }^{(\bowtie)}$, N. Olmaskhanov, N. Mirsamiev, and J. Mugisho \\ Department of Mineral Developing and Oil and Gas Engineering, \\ Engineering Academy, RUDN University, Moscow, Russia \\ simpleforfiza@mail.ru
}

\begin{abstract}
In this paper, a bench study of the pump-ejector system for simultaneous water and gas injection (SWAG) was conducted. For these purposes, a pump-ejector system stand was used. A differential pressure gauge was used to determine the gas flow at the ejector intake. According to the results of differential manometer calibrations, a new formula was obtained which reduces its inaccuracy to $1 \%$ at pressures below $0.6 \mathrm{MPa}$. In addition, according to the pressure-energy diagrams, it was determined that the gas injection with excess pressures in the ejector suction chamber significantly increases the efficiency of the pumping-ejector system overall.
\end{abstract}

Keywords: Liquid-gas ejector · Pump-ejector system $\cdot$ Liquid-gas mixture • SWAG

\section{Introduction}

Nowadays, a global trend of oil fields with increased residual oil saturation is observed (Drozdov and Drozdov 2012). There is also problem with associated petroleum gas (APG) utilization, which is not used rationally and is directed to gas flares. Currently, one of the most promising areas in oil production is the simultaneous water and gas injection (SWAG) technology. The definite advantage of the SWAG is the ability to use APG as a liquid-gas mixture compound. This method makes it possible to mount simple maintaining, reliable and efficient equipment, while providing a significant reduction in power consumption and increasing the SWAG efficiency (Drozdov et al. 2012).

\section{Methods and Approaches}

The investigation was conducted based on bench tests. The electric centrifugal pump and the liquid-gas ejector were used, which represent a single bench-model of the pump-ejector system. This stand is designed to investigate the characteristics of model ejectors, multistage centrifugal pumps and pump-ejector systems with liquid-gas mixtures using fresh water as a liquid, and air as a gas. The differential pressure gauge was used to determine the gas flow, with allowance for the excess pressure created in 
the suction chamber of the ejector. For operations at low pressures, the differential pressure gauge was pre-calibrated before.

\section{Results and Discussion}

Calibration of the differential manometer made it possible to clarify the existing formula for determining the gas flow rate at the ejector suction chamber at pressures below 0.6 MPa. The inaccuracy of the calculated values obtained by this method was less than $1 \%$. According to the results of the conducted research, the pressure-energy characteristics of the ejectors were constructed during gas suction by the liquid. There occurs a significant expansion of liquid-gas ejector working range by the injection of gas with excess pressure in the ejectors suction chamber as well as the areas of maximum values of injection coefficients and efficiency rates are shifted to higher region of operating pressure.

\section{Conclusions}

Further study of the pressure-energy characteristics behavior and the causes affecting this change will facilitate the increase of efficiency of technological processes which use the pumping-ejector systems.

\section{References}

Drozdov AN, Drozdov NA (2012) Laboratory researches of the heavy oil displacement from Russkoye field's core models at the SWAG injection and development of technological schemes of pump-ejecting systems for the water-gas mixtures delivering. In: SPE 157819, presented at the SPE heavy oil conference canada held in Calgary, Alberta, Canada, pp 12-14

Drozdov NA, Drozdov AN, Malyavko EA (2012) Investigation of SWAG injection and prospects of its implementation with the usage of pump-ejecting systems at existing oil-field infrastructure. In: SPE 160687, presented at the SPE Russian oil and gas exploration and production technical conference and exhibition held in Moscow, Russia, pp 16-18

Open Access This chapter is licensed under the terms of the Creative Commons Attribution 4.0 International License (http://creativecommons.org/licenses/by/4.0/), which permits use, sharing, adaptation, distribution and reproduction in any medium or format, as long as you give appropriate credit to the original author(s) and the source, provide a link to the Creative Commons license and indicate if changes were made.

The images or other third party material in this chapter are included in the chapter's Creative Commons license, unless indicated otherwise in a credit line to the material. If material is not included in the chapter's Creative Commons license and your intended use is not permitted by statutory regulation or exceeds the permitted use, you will need to obtain permission directly from the copyright holder. 